Document downloaded from:

http://hdl.handle.net/10251/52880

This paper must be cited as:

Camba, J.; Contero, M.; Johnson, M. (2014). Management of visual clutter in annotated 3D CAD models: A comparative study. Lecture Notes in Computer Science. 8518:405-416. doi:10.1007/978-3-319-07626-3_37.

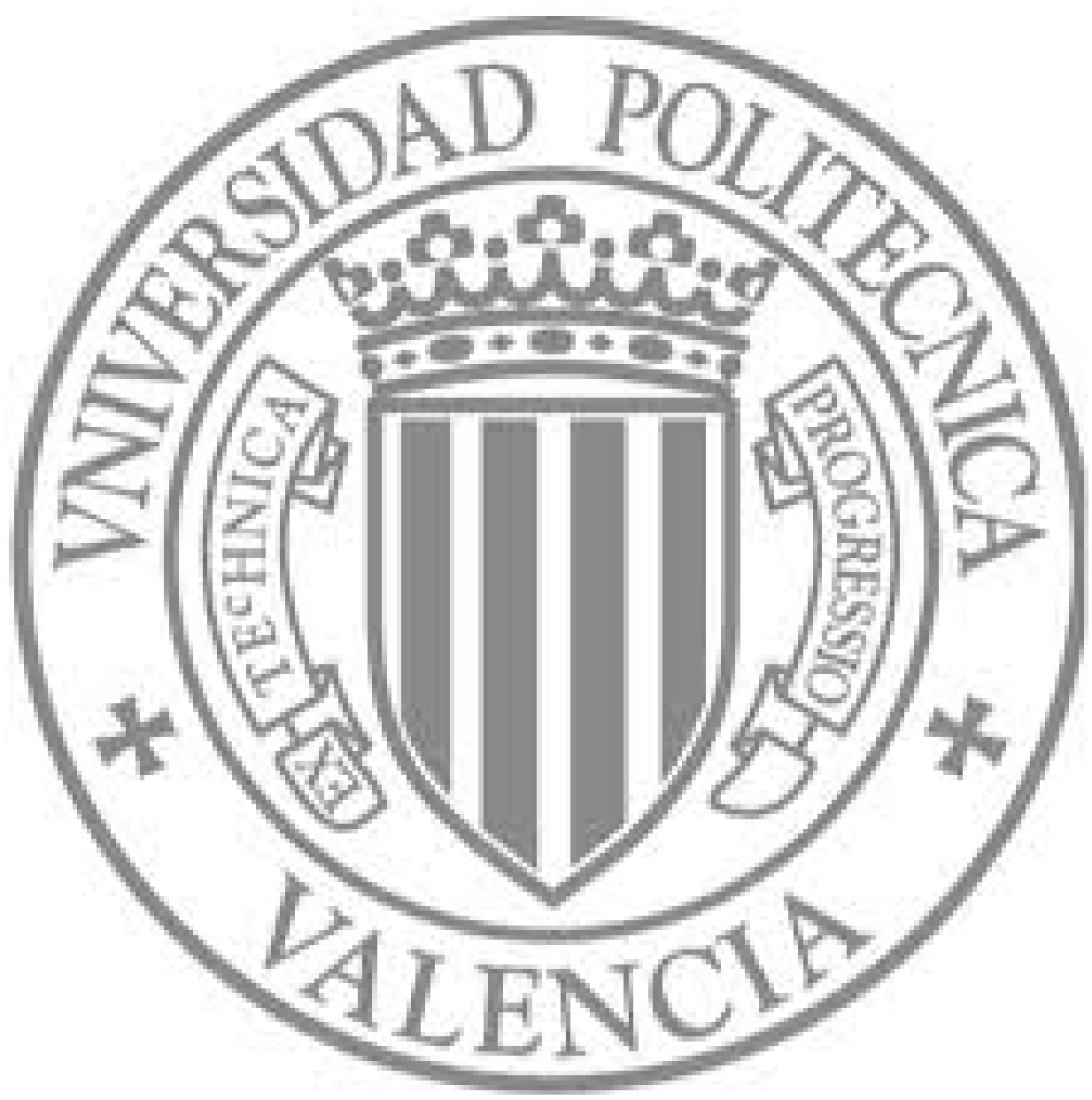

The final publication is available at

http://dx.doi.org/10.1007/978-3-319-07626-3_37

Copyright Springer Verlag (Germany) 


\title{
Management of Visual Clutter in Annotated 3D CAD Models: A Comparative Study
}

\author{
Jorge Camba $^{1}$, Manuel Contero ${ }^{2}$, Michael Johnson $^{3}$ \\ ${ }^{1}$ Engineering Design Graphics, Texas A\&M University, College Station, TX, USA \\ camba@tamu.edu \\ ${ }^{2}$ I3BH, Universitat Politècnica de València, València, Spain, 46010 \\ mcontero@upv.es \\ ${ }^{3}$ Dept. of Engineering Technology and Industrial Distribution, Texas A\&M University, \\ College Station, TX, USA \\ mdjohnson@tamu.edu
}

\begin{abstract}
The use of annotations in CAD models has been an active area of research because of their ability to connect design information to specific aspects of the model's geometry. The effectiveness of annotations is determined by the ability to clearly communicate information. However, annotations can quickly create clutter and confusion as they increase both in number and complexity. Consequently, efficient interaction and visualization mechanisms become crucial. Despite recent standardizations of procedures for the presentation of textual information in CAD models, no explicit guidelines are available as to how to make annotated models more readable and manageable. In this paper, we present the results of a comparative study of different mechanisms to manage visual clutter in annotated 3D CAD models and offer recommendations based on our findings. Our results show that even basic interaction mechanisms have a substantial impact on user's performance.
\end{abstract}

Keywords: visual clutter, annotated 3D models, CAD model interaction, design communication.

\section{Introduction}

Annotations are used in many different fields as a tool to provide comments, clarifications, descriptions, or interpretations about certain aspects of the object that is being annotated. In software development, for example, annotations have become a fundamental instrument in software quality and documentation activities [1-3]. They take the form of source code comments, which are used by programmers to describe what a program (or part of it) does and how it works. In the context of a CAD model, annotations are typically used to enhance the geometry with information that is valuable, but difficult to communicate otherwise (such as dimensions, instructions, or manufacturing information). 3D annotations are generally represented as blocks of text anchored to a specific part of the $3 \mathrm{D}$ model. They are pointers whose purpose is to draw the attention of the viewer to a specific area of the model. 
The use of 3D annotations in professional CAD environments has gained popularity in recent years, partly because of the development of standards for Digital Product Definition Data Practices [4, 5], which have formalized the way textual information is presented in a 3D model, and a growing interest in the Model-Based Engineering paradigm $[6,7]$, which puts $3 \mathrm{D}$ models at the center of the product development process. Most commercial CAD packages provide annotation tools that implement these standards, although few packages allow for active interaction with annotation information. Annotation functionalities are usually provided by the Product and Manufacturing Information (PMI) modules, which are used to convey information such as Geometric Dimensioning and Tolerancing (GD\&T), surface finish, and material specifications.

As 3D models become more comprehensive and serve as the central element of the Model-Based Engineering paradigm, both the complexity and number of annotations increase. Some authors have recently studied the potential of $3 \mathrm{D}$ annotations as carriers of design knowledge [8-10], which can drastically increase the amount of visual information on screen at any given time. This matter naturally demands mechanisms to support the interaction with the information as well as an efficient visualization of the annotations. Although none of the current engineering graphics standards provide explicit guidelines about managing 3D annotations, they do recommend the use of groups, layers, or views to make the model more manageable. Displaying all annotations in a complex model quickly creates clutter and confusion, which makes the use of the model impractical. From a usability standpoint, minimizing visual clutter in extensively annotated models is an essential factor to ensure effectiveness in terms of communication of information. In the following sections, we analyze the problem of visual clutter and its effects on design communication. We describe methods to improve user interaction with cluttered models and present the results of a series of comparative studies aimed at measuring the efficiency of such methods.

\section{Related Work}

\subsection{Annotations in Computer-Aided Design}

In the domain of Computer-Aided Design (CAD), an annotation can be defined as a piece of information connected to a specific part of the 3D model [11] and used to supplement the geometry. They are mechanisms to logically associate product information to geometric elements with the objective of making models comprehensive and reusable. Annotations are usually inserted as blocks of plain text, but they can also include multimedia content and hypertext.

Despite early support by major CAD packages, it was difficult for designers to work with annotations due to the proprietary and software-dependent nature of the tools, making it challenging to exchange information with other systems. In addition, the lack of a universal set of rules to manage the informational content created inconsistencies in annotation practices, which has had an effect in the adoption of 
annotations in industrial environments. With the advent of recent standards for Digital Product Definition [4, 5], most major commercial CAD packages have implemented Product and Manufacturing Information (PMI) modules with full support for textual annotations. Typically, the functionalities provided by PMI modules include basic manipulation of annotations (creation, edition, and deletion), definition and selection of annotation planes, visibility control, meta-data management, and predefined tools for creating standard dimensions and tolerances. In addition, most of these packages also provide low-level access to the annotation structures via their corresponding Application Programming Interfaces (API), which we have used as part of a previous study to implement an annotation manager tool [9].

Recently, some researchers have suggested extending the scope of the standard annotation to a complete and more comprehensive definition of the model (product and design data) $[8,12,13]$. Many authors agree on the potential of this mechanism to capture design decisions and mediate the interactions between designers [13-15].

Naturally, there are a number of challenges related to using 3D annotations as carriers of design knowledge. Some of these challenges include the development of efficient data structures to properly represent the annotation information, the creation of new interfaces to enter and retrieve data [11], and the development of syntax to unify annotation representations and formats [16]. From a user interaction standpoint, an ever increasing number of gradually more complex annotations in a model can quickly result in a cluttered workspace, which often creates confusion, frustration, and information overload in the user. Managing the problem of visual clutter in an efficient manner is a crucial aspect in the visualization of heavily annotated models that can ultimately affect engineering design communication [9].

\subsection{Visual Clutter}

The problem of visual clutter has a long history of research, particularly in the areas of cognitive psychology and human factors. However, the term is not easy to define. Intuitively, clutter can be understood as the phenomenon that occurs when one has too many items available at any given time. In fact, many methods related to clutter reduction in human-computer interaction involve the elimination of some of those items from the central part of the display [17, 18]. Some studies have shown, however, that clutter is not necessarily linked to the number of items, but to a state in which the items cause confusion in the user so they negatively affect performance $[19,20]$. Although too much information can certainly cause visual clutter, other factors such as perception (people do not always agree on the level of clutter), user experience, information relevance, and the level of information organization should also be considered [21].

Management of visual clutter is an important factor in user interface design and information visualization. When too much data (or when data is not well organized) is displayed on a too small area, the value of the information and the visualization as a whole diminishes, affecting usability [22]. Over the years, a vast amount of research has been done in the area of visual search [23-25] and clutter reduction. [26, 28]. Many of these techniques rely on the user driving the visualization to less cluttered states [18]. Other methods such as [26, 27] apply the principle of constant information 
density to always generate visualizations that are neither too cluttered nor too scattered. A variety of metrics to measure visual clutter has also been proposed [20, $21,29]$. Despite the large number of clutter reduction techniques, the diversity of the application domain makes it difficult to find one solution that can be applied to all problems. Furthermore, formal comparative and usability studies are scarce, particularly when compared to the diversity of solutions available [22].

In the domain of annotated 3D models, previous research has focused on algorithms for different annotation styles [30], annotation layouts to prevent occlusion [31], and annotations alignment to automatically arrange annotation information around the model $[32,33]$. However, no specific solutions are defined in current CAD annotations standards, and thus, no practical implementations are available in current PMI modules. Reducing clutter is left to the discretion of the user, who often chooses not to benefit from annotation tools because of the additional effort involved in this task. Clearly, there is a need for mechanisms to actively filter and manipulate the annotation information presented on screen (see Fig. 1).

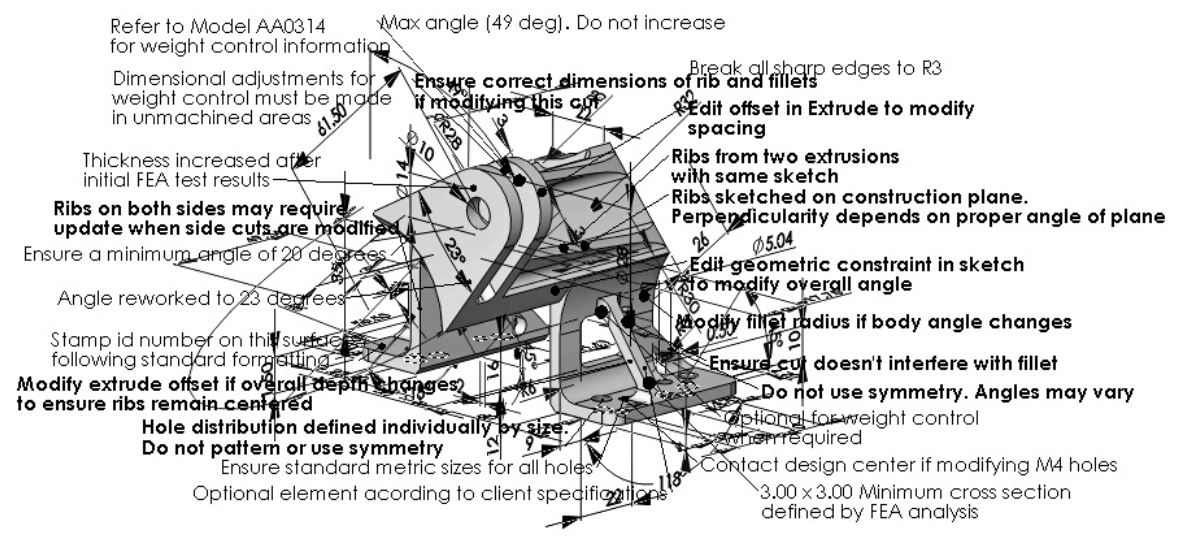

Fig. 1. Visual clutter caused by annotations

Filtering tools can contribute to reduce the amount of information on screen by showing or hiding specific model annotations based on user-defined criteria, such as date, feature, or a specific keyword. For example, users may select to only display the annotations associated to a particular surface of the model or created by a certain user.

An additional challenge regarding the use of model annotations, particularly the type of standard annotations provided by PMI modules in CAD environments, is related to their two-dimensional nature. Because annotations are essentially elements of plain text in 3D space, the user must use planes or views to host the annotations. As a result, some annotations may become visually unavailable when the user changes the viewpoint. Also, the creation and management of annotation planes and the distribution of annotations among these planes are ultimately the user's responsibility. According to current digital product definition standards [4, 5], the proper organization of model annotations in groups or annotation views is a recommended practice, although no specific rules are defined regarding how it should be implemented. 


\section{Software Prototype and Experimental Setup}

We present the results of a series of experiments that evaluate the performance of users in relation to the time required to find specific information in a heavily annotated CAD model. We measured and compared interactions when no managing mechanisms are in place (users must interact with all annotations on screen), when grouping and layering tools are available, when filtering methods are used (using a custom tool that the authors implemented for this purpose), and when interaction with the annotations is done via the model's design tree. We hypothesize that even the most basic mechanisms to manage annotations have a substantial impact on user's performance. Because of its availability and the familiarity of the participant students with the software, the commercial parametric modeling package SolidWorks ${ }^{\circledR}$ was used for all the activities.

The standard PMI module in SolidWorks ${ }^{\circledR}$ was used to test interactions via annotation groups. To evaluate user performance when using filtering tools and the model's design tree, our team developed a software tool that manages textual annotations. The tool was implemented as a plug-in for SolidWorks ${ }^{\circledR}$, allowing seamless integration with the CAD environment and direct interaction with the annotated 3D model.

Our software prototype implements a graphical user interface to filter annotations based on different criteria. All model annotations are also displayed in the software interface in a tabular form, facilitating navigation through annotation information. When a filter is applied, annotations that are not relevant become automatically hidden both in the model and in the annotation list in the prototype (see Fig. 2). Annotations become visible when the filter is reset or new criteria are defined.

Functionality for the interactive display of annotations based on the selection of model features in the design tree is also implemented by our tool. This function automatically recognizes the anchor point of the annotation to the geometry, and identifies the surface with its corresponding modeling feature.

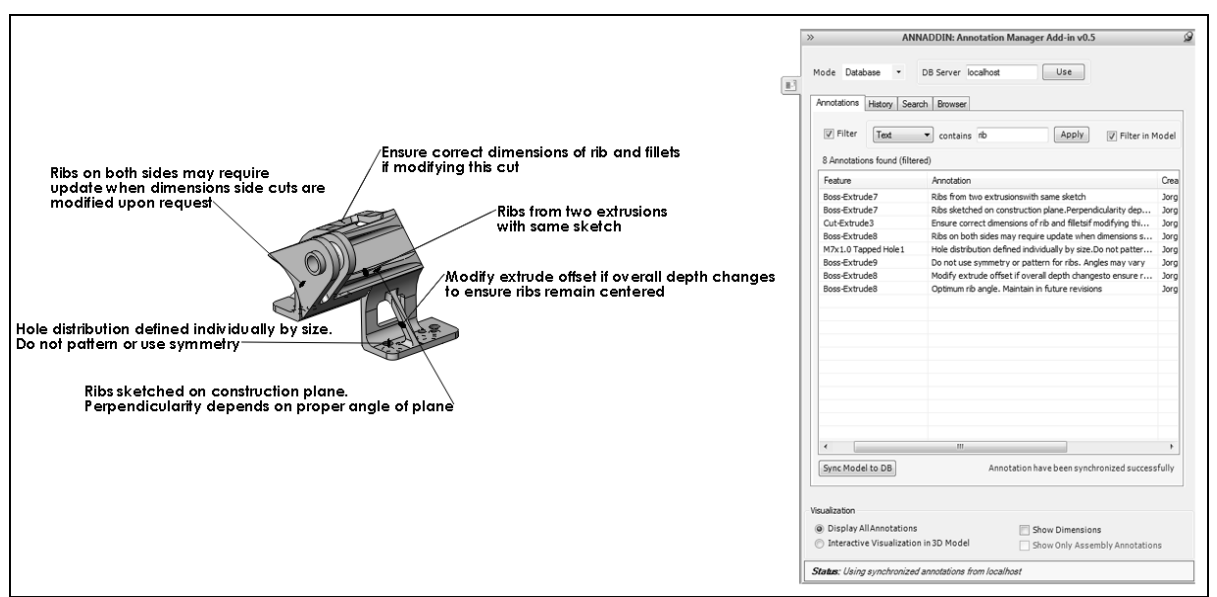

Fig. 2. Example of annotated model (left) and software prototype (right) after filtering annotations by keyword 


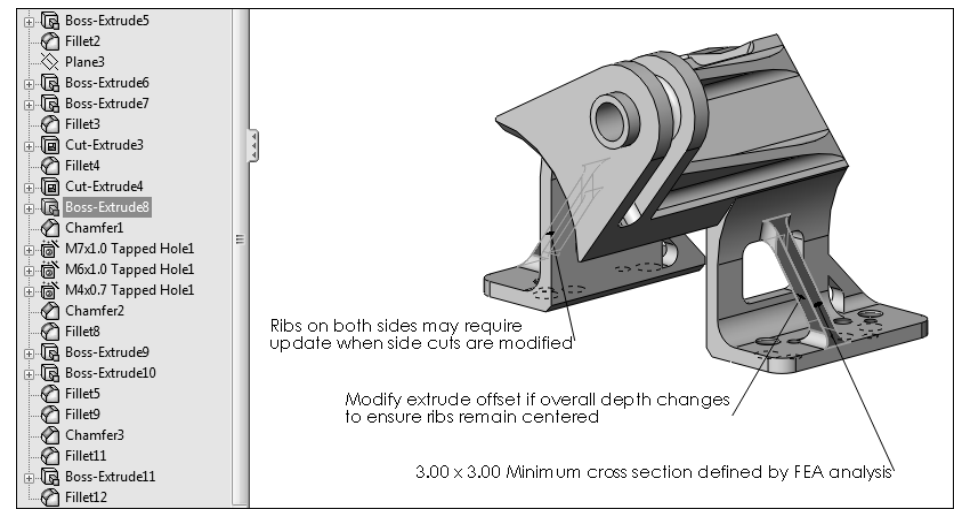

Figure 3. Example of interactive visualization of annotations. Only the $3 \mathrm{D}$ annotations connected to the feature that is selected in the design tree (left) are displayed in the model(right)

This method automatically shows or hides annotations based on the feature that is currently selected in the model's design tree (see Fig. 3).

\section{Experimental Procedure}

For our study, a total of 120 participants (divided into four groups of thirty) in a CAD laboratory environment were given an annotated model and asked to find specific model annotations with the answers to four questions. All participants were engineering students (mostly mechanical, aerospace, and industrial engineering majors) with at least one semester of training in engineering design graphics and practical experience using SolidWorks ${ }^{\circledR}$. Questions were presented one at a time. The first two questions (short answer) required participants to write their answer based on the information found in one particular annotation. The remaining two questions were presented in a multiple-choice format with four possible answers. They were formulated as design problems that required performing minor modification to the $3 \mathrm{D}$ model, but intentionally caused rebuild errors and unwanted effects in certain parts of the geometry. All possible answers described an adequate modeling approach to solve the design problem. Participants were asked to select the correct answer based on the information found in a specific group of model annotations. The time employed by participants to find the correct answer was recorded (starting from the time they received the question).

A CAD model with a total of thirty annotations deliberately arranged to create visual clutter was given to the participants. All dimensions were also visible (see Figure 1). The content of the annotations ranged from design and manufacturing information (i.e. Refer to model AA0314 for weight control information, Dimensional adjustments for weight control must be made in unmachined areas) to design intent and modeling procedures (i.e. Modify extrude offset if overall depth changes to ensure ribs remain centered, Hole distribution defined individually by size. Do not pattern or use symmetry). The annotations that are relevant to the questions of the study are 
shown in Table 1. In order to supplement instructions and clear any possible doubts, all questions were accompanied by illustrations of the 3D model, with specific areas highlighted. Such illustrations have been omitted in Table 1 for clarity.

Table 1. Questions and relevant annotations with explicit information to answer correctly.

\begin{tabular}{|c|c|c|c|}
\hline & Question & Answer & Annotations \\
\hline 1 & $\begin{array}{l}\text { What should you before modifying the } \\
\text { size of the M4 machine holes? }\end{array}$ & $\begin{array}{l}\text { Contact } \\
\text { Design Center }\end{array}$ & $\begin{array}{l}\text { Contact Design Center if } \\
\text { modifying M4 holes. }\end{array}$ \\
\hline 2 & $\begin{array}{l}\text { What is the range of acceptable angles } \\
\text { for the front face of the part? }\end{array}$ & $\begin{array}{l}\operatorname{Min}=20^{\circ} \\
\operatorname{Max}=27^{\circ}\end{array}$ & $\begin{array}{l}\text { Ensure min angle of } 20 \\
\text { degrees, Ensure max angle } \\
\text { of } 27 \text { degrees }\end{array}$ \\
\hline 3 & $\begin{array}{l}\text { What is the most effective procedure to } \\
\text { create a second rib on the model? } \\
\text { a) Mirror the first rib. } \\
\text { b) New sketch on surface and extrude. } \\
\text { c) Linear pattern using the original rib. } \\
\text { d) Use existing sketch of original rib to } \\
\text { create new extrusion at offset distance. }\end{array}$ & b) & $\begin{array}{l}\text { Do not use symmetry or } \\
\text { pattern for ribs. Angles may } \\
\text { vary. }\end{array}$ \\
\hline 4 & $\begin{array}{l}\text { When modifying the dimensions of the } \\
\text { ribs, errors occur in the model. What is } \\
\text { the procedure to solve these errors? } \\
\text { a) Reduce the height of the cut. } \\
\text { b) Move the cut down. } \\
\text { c) Increase the angle of the ribs. } \\
\text { d) The dimensions of the side ribs } \\
\text { cannot be changed. }\end{array}$ & b) & $\begin{array}{l}\text { Optimum rib angle. } \\
\text { Maintain in future versions. } \\
3.00 \times 3.00 \text { minimum cross } \\
\text { section defined by FEA } \\
\text { analysis. } \\
\text { Keep dimensions of the cut } \\
\text { constant. Modify position if } \\
\text { necessary. }\end{array}$ \\
\hline
\end{tabular}

Each group was randomly assigned an annotation mechanism, as shown in Table 2. Participants in group 3 were given a brief demonstration of the plug-in and were allowed a few minutes to familiarize themselves with the software. For participants in group 4, the prototype was preset to work in interactive mode. Therefore, there was no need to provide additional instructions.

Table 2. Annotation mechanisms and descriptions.

\begin{tabular}{|c|c|c|c|}
\hline Group & Mechanism & Description & Tool \\
\hline 1 & $\begin{array}{l}\text { All annotations } \\
\text { on screen }\end{array}$ & No annotation management tools & (Not Applicable) \\
\hline 2 & $\begin{array}{l}\text { Layers and } \\
\text { Grouping }\end{array}$ & $\begin{array}{l}\text { Annotations manually organized in } \\
\text { groups by a member of the research team }\end{array}$ & $\begin{array}{l}\text { PMI module: } \\
\text { standard annotation } \\
\text { views }\end{array}$ \\
\hline 3 & Filtering & $\begin{array}{l}\text { Annotations can be filtered based on } \\
\text { different criteria: content, feature, etc. }\end{array}$ & $\begin{array}{l}\text { Custom plug-in } \\
\text { developed in-house }\end{array}$ \\
\hline 4 & $\begin{array}{l}\text { Model's } \\
\text { Design Tree }\end{array}$ & $\begin{array}{l}\text { Interactive visualization using the } \\
\text { features available in the model's design } \\
\text { tree. }\end{array}$ & $\begin{array}{l}\text { Custom plug-in } \\
\text { developed in-house }\end{array}$ \\
\hline
\end{tabular}


For participants in group 2, our team decided to create six annotation groups and distribute the annotations among these groups. Groups were based on the following criteria: annotations that communicate restrictions to the model (i.e. Ensure standard metric sizes for all holes), previous changes performed to the model (i.e. Thickness increased after initial FEA test results), warnings about modeling actions (i.e. Hole distribution defined individually by size. Do not pattern or use symmetry), manufacturing notes (i.e. Break all sharp edges to R4), optional elements (i.e. Optional for weight control when required), and modeling decisions (i.e. Ribs sketched on construction plane. Perpendicularity depends on proper angle of plane). The time employed by our team to define and create the annotation groups in the model was not considered in our statistical analysis, although it obviously has a significant effect on efficiency.

\section{Results}

To statistically evaluate the effect of the various annotation mechanisms, the mean time to correctly answer each question in the various mechanism groups were compared using a two-sample t-test. These mean times are shown in Table 3 along with the standard deviation for each group and question. Group 1, with all annotations shown, was designated the control group; the mean answer time for each of the questions (and the total) for each other group was compared to group 1. These results are also shown in Table 3.

For all four questions and all three groups, the time required to correctly answer the question was statistically significantly lower $(\alpha=0.05)$ than that of group 1 . For all four questions, the time required was the least for group 4.

Table 3. Question completion time by group and statistical comparison to Group 1.

\begin{tabular}{cccccccc}
\hline & Group 1 & \multicolumn{2}{c}{ Group2 } & \multicolumn{2}{c}{ Group 3 } & \multicolumn{2}{c}{ Group 4 } \\
\hline & $M$ & $M$ & $t$ & $M$ & $t$ & $M$ & $t$ \\
& $(S D)$ & $(S D)$ & $p$ & $(S D)$ & $p$ & $(S D)$ & $p$ \\
\hline \multirow{2}{*}{ Question 1 } & 212.6 & 82.2 & 9.89 & 65.5 & 11.10 & 54.0 & 12.10 \\
& $(70.4)$ & $(16.0)$ & $<0.001$ & $(17.7)$ & $<0.001$ & $(14.0)$ & $<0.001$ \\
& & & & & & & \\
Question 2 & 131.3 & 77.0 & 12.48 & 72.5 & 12.91 & 69.5 & 13.36 \\
& $(77.0)$ & $(13.3)$ & $<0.001$ & $(15.2)$ & $<0.001$ & $(15.8)$ & $<0.001$ \\
& & & & & & & \\
Question 3 & 310.2 & 168.7 & 21.90 & 169.4 & 14.83 & 153.3 & 21.17 \\
& $(168.7)$ & $(26.5)$ & $<0.001$ & $(46.4)$ & $<0.001$ & $(33.1)$ & $<0.001$ \\
& & & & & & & \\
Question 4 & 663.1 & 363.8 & 16.68 & 370.9 & 15.16 & 320.6 & 18.39 \\
& $(362.8)$ & $(30.1)$ & $<0.001$ & $(48.4)$ & $<0.001$ & $(40.0)$ & $<0.001$ \\
& & & & & & & \\
Total & 1317.1 & 690.7 & 21.71 & 678.3 & 21.02 & 597.4 & 23.83 \\
& $(690.7)$ & $(50.2)$ & $<0.001$ & $(72.4)$ & $<0.001$ & $(70.1)$ & $<0.001$ \\
\hline
\end{tabular}


The total time required to correctly answer all for questions was compared for each of the alternative annotation mechanisms. These results are shown in Table 4. There was no statistically significant difference in the total time required to answer the questions between the mechanisms used for groups 2 and 3. Only Question 1 showed a statistically significant difference between groups 2 and $3(t=3.83 ; p=<0.001)$. In the comparison between groups 2 and 4, only Question 3 did not show a statistically significant difference $(t=1.99 ; p=0.052)$. In the comparison of groups 3 and 4 , Questions $2(t=0.76 ; p=0.447)$ and $3(t=1.54 ; p=0.128)$ did not show statistically significant differences between the two mechanisms.

Although group 4 was the best performer in terms of time to find specific annotations, it cannot be concluded that the interaction mechanism used by this group is the most efficient in terms of communicating information. In a situation where one or more features in the design tree are left unexplored, the potential annotations connected to them will never be displayed. Therefore, some relevant information may never reach the user. Nevertheless, interactive visualization via the design tree has proven successful in reducing visual clutter on screen.

Table 4. Total time for all questions data comparison.

\begin{tabular}{cccc}
\hline & Group 2 & Group 3 & Group 4 \\
\hline & $t$ & $t$ & $t$ \\
& $p$ & $p$ & $p$ \\
\hline \multirow{2}{*}{ Group 1 } & 21.71 & 21.02 & 23.83 \\
& $<0.001$ & $<0.001$ & $<0.001$ \\
& & 0.77 & 5.93 \\
Group 2 & 0.444 & $<0.001$ \\
& & & 4.40 \\
Group 3 & & $<0.001$ \\
\hline
\end{tabular}

We observed a tendency in a large number of participants in group 4 (interaction using the design tree) to click and select surfaces directly in the 3D model (as opposed to selecting the feature in the design tree) to try to activate the associated annotations. When only one surface of the model is selected, the current version of our prototype will only display the annotations that are directly anchored to that surface. However, when a feature in the design tree is selected, the tool will show all the annotations connected to any of the surfaces in that feature. In our experiments, a number of participants did not realize this behavior until after several trials. These actions could explain some of the slower times recorded for this group, particularly in the first activity. On the positive side, these results can also be interpreted as indicators of a more intuitive behavior expected by the user, which can be an important factor to consider in future implementations of our prototype. 


\section{Conclusions and Future Work}

Our results expose the limitations of current techniques for managing the visual clutter created by $3 \mathrm{D}$ annotations (even in models with a relatively small number of annotations) and confirm the need for intuitive methods to browse and filter annotations. As originally anticipated, users that were asked to retrieve information from models with no visualization or filtering tools performed statistically significantly worse than users with access to annotation management mechanisms. On the other hand, filtering and interactive navigation based on the model's design tree are faster and more efficient techniques than organizing the annotations in groups or annotation views (as suggested by current standards), especially if time required to create the groups and properly distribute the annotations within these groups are taken into consideration.

For our study, we implemented a software prototype that enhances the functionalities of standard PMI modules in CAD packages, reduces visual clutter, and provides users with a more intuitive and efficient mechanism to interact with annotations. In the future, we plan to explore and evaluate other mechanisms and interfaces that can be applied to CAD environments. We are interested in analyzing the effects of color and other perceptual elements such as annotation size, style, and patterns to define more sophisticated strategies that support visual searches. Finally, new techniques to simplify the creation and distribution of annotations among layers and groups are also desirable. Despite the reduction of clutter observed in our study when using annotation layers (group 2), the process of organizing information in groups has proven tedious and time consuming. In addition, we could have created different groups based on alternative organization criteria, which could have impacted user performance and thus provide different outcomes. Ideally, a system that performed this task automatically or semi-automatically would potentially provide enormous benefits to users of annotated CAD models.

Finally, our team observed that some of the difficulties experienced by participants were related to the two-dimensional nature of standard CAD annotations. Even after filtering annotations or hiding unnecessary groups, user interaction was often not intuitive, as some information was not visually available when the CAD model was rotated. This effect was noticeable when the orientation of the annotation plane changed drastically with respect to the viewing plane. Moving from a pure twodimensional annotation structure to a system where annotations are readable from any angle regardless of the orientation of the CAD model and the position of the annotation plane could significantly improve visualization.

\section{References}

1. Kajko-Mattsson, M.: The State of Documentation Practice within Corrective Maintenance. In: IEEE International Conference on Software Maintenance, pp. 354-363. IEEE Press, New York (2001)

2. Van De Vanter, M.L.: The Documentary Structure of Source Code. Information and Software Technology. 44, 767-782 (2002) 
3. Haouari, D., Sahraoui, H., Langlais, P.: How Good is Your Comment? A Study of Comments in Java Programs. In: International Symposium on Empirical Software Engineering and Measurement, pp. 137-146. IEEE Press, New York (2011)

4. ASME Y14.41-2012 Digital Product Definition Data Practices. The American Society of Mechanical Engineers, New York, 2012

5. ISO 16792:2006 Technical Product Documentation - Digital Product Definition Data Practices. Organisation Internationale de Normalisation, Genève, Suisse, 2006.

6. Boehm, B., Bayuk, J., Desmukh, A., Graybill, R., Lane, J.A., Levin, A., et al.: Systems 2020 Strategic Initiative. DoD Systems Engineering Research Center, Technical Report, SERC-2010-TR-009 (2010)

7. Quintana, V, Rivest, L, Pellerin, R.: Measuring and Improving the Process of Engineering Change Orders in a Model-Based Definition Context. International Journal of Product Lifecycle Management. 6(2), 138-160 (2012)

8. Alducin-Quintero, G., Rojo, A., Plata, F., Hernández, A., Contero, M.: 3D Model Annotation as a Tool for Improving Design Intent Communication: A Case Study on its Impact in the Engineering Change Process. In: ASME International Design Engineering Technical Conferences \& Computers and Information in Engineering Conference, pp. 349-356. ASME, New York (2012)

9. Dorribo-Camba, J., Alducin-Quintero, G, Perona, P., Contero, M.: Enhancing Model Reuse through 3D Annotations: A Theoretical Proposal for an Annotation-Centered Design Intent and Design Rationale Communication. In: ASME International Mechanical Engineering Congress \& Exposition. ASME, New York (2013)

10. Ding, L., Davies, D., McMahon, C.: Sharing Information throughout a Product Lifecycle via Markup of Product Models. In: ASME International Design Engineering Technical Conferences \& Computers and Information in Engineering Conference. pp. 1267-1275, ASME, New York (2008)

11. Ding, L., Ball, A., Patel, M., Matthews, J., Mullineux, G.: Strategies for the Collaborative Use of CAD Product Models. In: Proceedings of ICED 09, 17th International Conference on Engineering Design, vol. 8, pp. 123-134 (2009)

12. Ding, L., Davies, D., McMahon, C.A.: The Integration of Lightweight Representation and Annotation for Collaborative Design Representation. Research in Engineering Design. 20(3), 185-200 (2009)

13. Boujut, J.F., Dugdale, J.: Design of a 3D Annotation Tool for Supporting Evaluation Activities in Engineering Design. Cooperative Systems Design. 6, 1-8 (2006)

14. Bracewell, R.H., Wallace, K.M.: A Tool for Capturing Design Rationale. In: 14th International Conference on Engineering Design. Paper no. DS31_1437FPB (2003)

15. Patel, M., Ball, A., Ding, L.: Curation and Preservation of CAD Engineering Models in Product Lifecycle Management. In: $14^{\text {th }}$ International Conference on Virtual Systems and Multimedia Dedicated to Digital Heritage, pp. 59-66 (2008)

16. Li, C., McMahon, C., Newnes, L.: Annotation in Product Lifecycle Management: A Review of Approaches. In: ASME International Design Engineering Technical Conferences and Computers and Information in Engineering Conference, pp. 797-806, (2009)

17. Ahlberg, C., Shneiderman, B.: Visual Information Seeking: Tight Coupling of Dynamic Query Filters with Starfield Displays. In: SIGCHI Conference on Human Factors in Computing Systems. pp. 313-317 (1994)

18. Fishkin, K., Stone, M.C.: Enhanced Dynamic Queries via Movable Filters. In: SIGCHI Conference on Human Factors in Computing Systems. pp. 415-420 (1995) 
19. Noyes, L.: The Positioning of Type on Maps: The Effect of Surrounding Material on Word Recognition Time. Human Factors. 22(3), 353-360 (1980)

20. Rosenholtz, R., Li, Y., Mansfield, J., Jin, Z.: Feature Congestion: A Measure of Display Clutter. In: SIGCHI Conference on Human Factors in Computing Systems. pp. 761-770 (2005)

21. Tufte, E.R.: The Visual Display of Quantitative Information. Graphics Press, Cheshire, CT, (1983)

22. Ellis, G., Dix, A.: A Taxonomy of Clutter Reduction for Information Visualisation. IEEE Transactions on Visualization and Computer Graphics. 13(6), 1216-1223 (2007)

23. Wolfe, J.M.: Guided Search 2.0: A Revised Model of Visual Search. Psychonomic Bulletin \& Review, 1(2), 202-238 (1994)

24. Palmer, J.: Set-size Effects in Visual Search: the Effect of Attention is Independent of the Stimulus for Simple Tasks. Vision Research, 34, 1703-1721 (1994)

25. Rosenholtz, R.: Search asymmetries? What search asymmetries? Perception \& Psychophysics, 63(3), 476-489 (2001)

26. Woodruff, A., Landay, J., Stonebraker, M.: Constant Information Density in Zoomable Interfaces. In: Working Conference on Advanced Visual Interfaces, pp. 57-65 (1998)

27. Ellis, G., Bertini, E., Dix, A.: The Sampling Lens: Making Sense of Saturated Visualisations. In: Extended Abstracts on Human Factors in Computing Systems. pp. 1351-1354 (2005)

28. Ellis, G., Dix, A.: Enabling Automatic Clutter Reduction in Parallel Coordinate Plots. IEEE Transactions on Visualization and Computer Graphics, 12(5), 717-723 (2006)

29. Frank, A.U., Timpf, S.: Multiple Representations for Cartographic Objects in a Multi-scale Tree - An Intelligent Graphical Zoom. Computers \& Graphics, 18(6), 823-829 (1994)

30. Cipriano, G., Gleicher, M.: Text Scaffolds for Effective Surface Labeling. IEEE Transactions on Visualization and Computer Graphics. 14(6), 1675-1682 (2008)

31. Stein, T., Décoret, X.: Dynamic Label Placement for Improved Interactive Exploration. In: $6^{\text {th }}$ International Symposium on Non-Photorealistic Animation and Rendering, pp. 15-21 (2008)

32. Ali, K., Hartmann, K., Strothotte, T.: Label Layout for Interactive 3D Illustrations. Journal of WSCG 13(1), pp. 1-8 (2005)

33. Götzelmann, T., Hartmann, K., Strothotte, T.: Agent-Based Annotation of Interactive 3D Visualizations. In: Butz, A., Fisher, B., Krüger, A. Olivier, P. (Eds.) Smart Graphics 2006. LNCS, vol. 4073, pp. 24-35. Springer, Heidelberg (2006) 